\title{
Dirac quantization of membrane winding uniformly in time dependent orbifold
}

\author{
Przemysław Małkiewicz and Włodzimierz Piechocki \\ Theoretical Physics Department, Institute for Nuclear Studies, \\ Hoża 69, 00-681 Warszawa, Poland \\ E-mail: pmalk@fuw.edu.pl, piech@fuw.edu.pl
}

\begin{abstract}
We present quantum theory of a membrane propagating in the vicinity of a time dependent orbifold singularity. The dynamics of a membrane, with the parameters space topology of a torus, winding uniformly around compact dimension of the embedding spacetime is mathematically equivalent to the dynamics of a closed string in a flat FRW spacetime. The construction of the physical Hilbert space of a membrane makes use of the kernel space of self-adjoint constraint operators. It is a subspace of the representation space of the constraints algebra. There exist non-trivial quantum states of a membrane evolving across the singularity.
\end{abstract}

KeYwords: p-branes, Field Theories in Higher Dimensions, Spacetime Singularities 


\section{Contents}

1 Introduction 1

2 Hamiltonian 2

3 Algebra of constraints 4

4 Meaning of the constraints 5

5 Representations $\quad 6$

$\begin{array}{lll}5.1 & \text { Representation based on a single field } & 7\end{array}$

$\begin{array}{lll}5.1 .1 & \text { Hilbert space } & 7\end{array}$

$\begin{array}{lll}5.1 .2 & \text { Representation of generator } & 7\end{array}$

5.1.3 Solving the constraint 8

$\begin{array}{ll}\text { 5.1.4 Interpretation of solutions } & 9\end{array}$

5.2 Representation based on phase space functions 9

5.2.1 Hilbert space $\quad 9$

$\begin{array}{ll}\text { 5.2.2 Solving the constraint } & 11\end{array}$

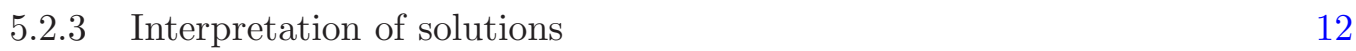

$\begin{array}{lll}5.3 & \text { Remarks on representations of observables } & 12\end{array}$

6 Conclusions 13

$\begin{array}{ll}\text { A Local form of the constraints algebra } & 14\end{array}$

\section{Introduction}

In the cyclic universe scenario [1,2] an evolution of the universe consists of the sequence of classical and quantum phases. One examines the possibility of describing each of quantum phases in terms of quantum elementary objects in higher dimensional $(d>4)$ compactified Milne, CM, space. The CM space includes a cosmological singularity which consists of bigcrunch and big-bang epochs [3]. Propagation of elementary objects across the singularity is the main concern.

A reasonable model of spacetime with the cosmological singularity should allow for propagation of a quantum p-brane (i.e., particle, string, membrane,...) from the presingularity to the post-singularity epoch. If the quantum $p$-brane cannot go through the cosmological singularity, the cyclic evolution cannot be realized. In our previous papers we have examined the evolution of a particle $[4,5]$ and a string $[6,7]$ across the singularity. A model of the quantum phase in the above sense seems to be well defined. 
The case of a membrane is technically more complicated because functions describing membrane dynamics depend on three variables. The Hamilton equations for these functions constitute a system of coupled non-linear equations in higher dimensional phase space. The Cauchy problem for membranes has been examined so far only in ambient Lorentz manifolds [8]. Owing to this complexity, we only try to identify some non-trivial membrane states which propagate through the cosmological singularity.

The first-class constraints describing membrane dynamics are generators of gauge transformations in the phase space of the system and come from the reparametrization invariance of an action integral. The goal of the present paper is the construction of a quantum theory of a membrane winding around compact dimension of CM space. The Hilbert space of a quantum membrane is constructed by making use of the kernel space of the constraints $[10,11]$.

Motivation for the present work is strictly cosmological, in spite of the fact that the model has been inspired by string/M theory [3]. We do not intend to address in this paper any problem of the $M$ theory. Getting inside into the cosmological problem is our main concern. However, our quantization seems to be a novelty in the field of strings and it might be extended beyond considered cosmological model.

In this paper we consequently use the Dirac method for quantizing the Hamiltonian systems with constraints, which in principle may be applied to the quantization of any $p$-brane propagating in curved spacetime. Our method is different from the one used in the textbooks on string theory (see, e.g. [12]). This is probably why the critical dimension of spacetime does not occur in our results.

The paper is organized as follows: section 2 deals with essentials of the classical formulation of the system. In section 3 we define an algebra of Hamiltonian constraints of a membrane. In section 4 we interpret constraints as generators for coordinate transformations. Section 5 concerns finding the representation of the constraints algebra. We consider a toy model to get some insight into the problem by using a single field. Then, we use some fields defined on the phase space of the membrane in the background space to construct the physical Hilbert space. We conclude in the last section.

\section{Hamiltonian}

The Polyakov action for a test $p$-brane embedded in a background spacetime with metric $g_{\tilde{\mu} \tilde{\nu}}$ has the form

$$
S_{p}=-\frac{1}{2} \mu_{p} \int d^{p+1} \sigma \sqrt{-\gamma}\left(\gamma^{a b} \partial_{a} X^{\tilde{\mu}} \partial_{b} X^{\tilde{\nu}} g_{\tilde{\mu} \tilde{\nu}}-(p-1)\right),
$$

where $\mu_{p}$ is a mass per unit $p$-volume, $\left(\sigma^{a}\right) \equiv\left(\sigma^{0}, \sigma^{1}, \ldots, \sigma^{p}\right)$ are $p$-brane worldvolume coordinates, $\gamma_{a b}$ is the $p$-brane worldvolume metric, $\gamma:=\operatorname{det}\left[\gamma_{a b}\right], \quad\left(X^{\tilde{\mu}}\right) \equiv\left(X^{\mu}, \Theta\right) \equiv$ $\left(T, X^{k}, \Theta\right) \equiv\left(T, X^{1}, \ldots, X^{d-1}, \Theta\right)$ are the embedding functions of a $p$-brane, i.e. $X^{\tilde{\mu}}=$ $X^{\tilde{\mu}}\left(\sigma^{0}, \ldots, \sigma^{p}\right)$, in $d+1$ dimensional background spacetime. 
It has been found [16] that the total Hamiltonian, $H_{T}$, corresponding to the action (2.1) is the following

$$
H_{T}=\int d^{p} \sigma \mathcal{H}_{T}, \quad \mathcal{H}_{T}:=A C+A^{i} C_{i}, \quad i=1, \ldots, p
$$

where $A=A\left(\sigma^{a}\right)$ and $A^{i}=A^{i}\left(\sigma^{a}\right)$ are any functions of $p$-volume coordinates,

$$
\begin{array}{r}
C:=\Pi_{\tilde{\mu}} \Pi_{\tilde{\nu}} g^{\tilde{\mu} \tilde{\nu}}+\mu_{p}^{2} \operatorname{det}\left[\partial_{a} X^{\tilde{\mu}} \partial_{b} X^{\tilde{\nu}} g_{\tilde{\mu} \tilde{\nu}} \approx 0,\right. \\
C_{i}:=\partial_{i} X^{\tilde{\mu}} \Pi_{\tilde{\mu}} \approx 0,
\end{array}
$$

where $\Pi_{\tilde{\mu}}$ are the canonical momenta corresponding to $X^{\tilde{\mu}}$, and where the symbol ' $\approx$ ' denotes 'weakly zero' in the sense of Dirac [10]. Equations (2.3) and (2.4) define the first-class constraints of the system.

The Hamilton equations are

$$
\dot{X}^{\tilde{\mu}} \equiv \frac{\partial X^{\tilde{\mu}}}{\partial \tau}=\left\{X^{\tilde{\mu}}, H_{T}\right\}, \quad \dot{\Pi}_{\tilde{\mu}} \equiv \frac{\partial \Pi_{\tilde{\mu}}}{\partial \tau}=\left\{\Pi_{\tilde{\mu}}, H_{T}\right\}, \quad \tau \equiv \sigma^{0},
$$

where the Poisson bracket is defined by

$$
\{\cdot, \cdot\}:=\int d^{p} \sigma\left(\frac{\partial \cdot}{\partial X^{\tilde{\mu}}} \frac{\partial \cdot}{\partial \Pi_{\tilde{\mu}}}-\frac{\partial \cdot}{\partial \Pi_{\tilde{\mu}}} \frac{\partial \cdot}{\partial X^{\tilde{\mu}}}\right) .
$$

In what follows we restrict our considerations to the compactified Milne, CM, space. The CM space is one of the simplest models of spacetime implied by string/M theory [3]. Its metric is defined by the line element

$$
d s^{2}=-d t^{2}+d x^{k} d x_{k}+t^{2} d \theta^{2}=\eta_{\mu \nu} d x^{\mu} d x^{\nu}+t^{2} d \theta^{2}=g_{\tilde{\mu} \tilde{\nu}} d x^{\tilde{\mu}} d x^{\tilde{\nu}},
$$

where $\eta_{\mu \nu}$ is the Minkowski metric, and $\theta$ parameterizes a circle. Orbifolding $\mathbb{S}^{1}$ to a segment $\mathbb{S}^{1} / \mathbb{Z}_{2}$ gives the model of spacetime in the form of two planes which collide and re-emerge at $t=0$. Such model of spacetime has been used in [1,2]. Our results do not depend on the choice of topology of the compact dimension.

In our previous paper [6] and in the present one we analyze the dynamics of a $p$-brane which is winding uniformly around the $\theta$-dimension. For the $p$-brane in such a state, we identify the $p$-th worldvolume coordinate with the compact dimension $\theta$ and impose the condition that the state of the $p$-brane does not depend on this coordinate, i.e.

$$
\sigma^{p}=\theta=\Theta \quad \text { and } \quad \partial_{\theta} X^{\mu}=0=\partial_{\theta} \Pi_{\mu}
$$

which leads to

$$
\frac{\partial}{\partial \theta}\left(X^{\tilde{\mu}}\right)=(0, \ldots, 0,1) \quad \text { and } \quad \frac{\partial}{\partial \tau}\left(X^{\tilde{\mu}}\right)=\left(\dot{T}, \dot{X}^{k}, 0\right) .
$$

The conditions (2.8) reduce (2.3)-(2.6) to the form in which the canonical pair $\left(\theta, \Pi_{\theta}\right)$ does not occur [16]. 


\section{Algebra of constraints}

In the case of a winding uniformly membrane applying the condition (2.8) to the formulae (2.3) and (2.4) leads to the two constraints

$$
\begin{aligned}
C & =\Pi_{\mu}(\tau, \sigma) \Pi_{\nu}(\tau, \sigma) \eta^{\mu \nu}+\kappa^{2} T^{2}(\tau, \sigma) \dot{X}^{\mu}(\tau, \sigma) \dot{X}^{\nu}(\tau, \sigma) \eta_{\mu \nu} \approx 0, \\
C_{1} & =\dot{X}^{\mu}(\tau, \sigma) \Pi_{\mu}(\tau, \sigma) \approx 0, \quad C_{2}=0,
\end{aligned}
$$

where $\dot{X}^{\mu}:=\partial X^{\mu} / \partial \sigma, \quad \sigma:=\sigma^{1}, \kappa:=\theta_{0} \mu_{2}$, and where $\theta_{0}:=\int d \theta$.

To examine the algebra of constraints we 'smear' the constraints as follows

$$
\check{A}:=\int_{-\pi}^{\pi} d \sigma f(\sigma) A\left(X^{\mu}, \Pi_{\mu}\right), \quad f \in\left\{C^{\infty}[-\pi, \pi] \mid f^{(n)}(-\pi)=f^{(n)}(\pi)\right\} .
$$

The Lie bracket is defined as

$$
\{\check{A}, \check{B}\}:=\int_{-\pi}^{\pi} d \sigma\left(\frac{\partial \check{A}}{\partial X^{\mu}} \frac{\partial \check{B}}{\partial \Pi_{\mu}}-\frac{\partial \check{A}}{\partial \Pi_{\mu}} \frac{\partial \check{B}}{\partial X^{\mu}}\right) .
$$

The constraints in an integral form satisfy the algebra

$$
\begin{aligned}
\left\{\check{C}\left(f_{1}\right), \check{C}\left(f_{2}\right)\right\} & =\check{C}_{1}\left(4 \kappa^{2} T^{2}\left(f_{1} \dot{f}_{2}-\dot{f}_{1} f_{2}\right)\right), \\
\left\{\check{C}_{1}\left(f_{1}\right), \check{C}_{1}\left(f_{2}\right)\right\} & =\check{C}_{1}\left(f_{1} \dot{f}_{2}-\dot{f}_{1} f_{2}\right), \\
\left\{\check{C}\left(f_{1}\right), \check{C}_{1}\left(f_{2}\right)\right\} & =\check{C}\left(f_{1} \dot{f}_{2}-\dot{f}_{1} f_{2}\right) .
\end{aligned}
$$

Equations (3.5)-(3.7) demonstrate that $C$ and $C_{1}$ are first-class constraints because the Poisson algebra closes. However, it is not a Lie algebra because the factor $T^{2}$ is not a constant, but a function on phase space. Little is known about representations of such type of an algebra. Similar mathematical problem occurs in general relativity (see, e.g. [13]).

The smearing (3.3) of constraints helps to get the closure of the algebra in an explicit form. A local form of the algebra includes the Dirac delta so the algebra makes sense but in the space of distributions (see appendix A for more details). It seems that such an arena is inconvenient for finding a representation of the algebra which is required in the quantization procedure.

The original algebra of constraints may be rewritten in a tractable form by making use of the redefinitions

$$
C_{ \pm}:=\frac{C \pm C_{1}}{2}
$$

where

$$
C:=\frac{\text { original } C}{2 \kappa T}, \quad C_{1}:=\text { original } C_{1},
$$

where 'original' means defined by (3.1) and (3.2). The new algebra reads

$$
\begin{aligned}
& \left\{\check{C}_{+}(f), \check{C}_{+}(g)\right\}=\check{C}_{+}(f \dot{g}-g f), \\
& \left\{\check{C}_{-}(f), \check{C}_{-}(g)\right\}=\check{C}_{-}(f \dot{g}-g \dot{f}), \\
& \left\{\check{C}_{+}(f), \check{C}_{-}(g)\right\}=0 .
\end{aligned}
$$


The redefined algebra is a Lie algebra.

The redefinition (3.9) seems to be a technical trick without a physical interpretation. In what follows we show that it corresponds to the specification of the winding zero-mode state of a membrane not at the level of the phase space, but at the level of an action integral.

The Nambu-Goto action for a membrane in the CM space reads

$$
\begin{aligned}
S_{N G} & =-\mu_{2} \int d^{3} \sigma \sqrt{-\operatorname{det}\left(\partial_{a} X^{\mu} \partial_{b} X^{\nu} g_{\mu \nu}\right)} \\
& =-\mu_{2} \int d^{3} \sigma \sqrt{-\operatorname{det}\left(-\partial_{a} T \partial_{b} T+T^{2} \partial_{a} \Theta \partial_{b} \Theta+\partial_{a} X^{k} \partial_{b} X_{k}\right)}
\end{aligned}
$$

where $\left(T, \Theta, X^{k}\right)$ are embedding functions of the membrane corresponding to the spacetime coordinates $\left(t, \theta, x^{k}\right)$ respectively.

An action $S_{N G}$ in the lowest energy winding mode, defined by (2.9), has the form

$$
\begin{aligned}
S_{N G} & =-\mu_{2} \theta_{0} \int d^{2} \sigma \sqrt{-T^{2} \operatorname{det}\left(-\partial_{a} T \partial_{b} T+\partial_{a} X^{k} \partial_{b} X_{k}\right)} \\
& =-\mu_{2} \theta_{0} \int d^{2} \sigma \sqrt{-\operatorname{det}\left(\partial_{a} X^{\alpha} \partial_{b} X^{\beta} \widetilde{g}_{\alpha \beta}\right)} .
\end{aligned}
$$

where $a, b \in\{0,1\}$ and $\widetilde{g}_{\alpha \beta}=T \eta_{\alpha \beta}$. Thus, the propagation of a membrane in this special mode is equivalent to the evolution of a string in the spacetime with dimension $d$ (while $d+1$ was the original one), which is now a flat Friedmann-Robertson-Walker spacetime, $d s^{2}=t \eta_{\alpha \beta}$.

One can verify that the Hamiltonian corresponding to the string action (3.16) has the form

$$
H_{T}=\int d \sigma \mathcal{H}_{T}, \quad \mathcal{H}_{T}:=A C+A^{1} C_{1}
$$

where

$$
C:=\frac{1}{2 \mu_{2} \theta_{0} T} \Pi_{\alpha} \Pi_{\beta} \eta^{\alpha \beta}+\frac{\mu_{2} \theta_{0}}{2} T \partial_{a} X^{\alpha} \partial_{b} X^{\beta} \eta_{\alpha \beta} \approx 0, \quad C_{1}:=\partial_{\sigma} X^{\alpha} \Pi_{\alpha} \approx 0,
$$

and $A=A(\tau, \sigma)$ and $A^{1}=A^{1}(\tau, \sigma)$ are any regular functions. Therefore (3.18) and (3.9) coincide, which gives an interpretation for the redefinition of the constraints.

\section{Meaning of the constraints}

An action integral of a string is invariant with respect to smooth and invertible maps of worldsheet coordinates

$$
(\tau, \sigma) \rightarrow\left(\tau^{\prime}, \sigma^{\prime}\right)
$$

These diffeomorphisms considered infinitesimally form an algebra of local fields $-\epsilon(\tau, \sigma) \partial_{\tau}$ and $-\eta(\tau, \sigma) \partial_{\sigma}$ (we refer to their actions on the fields as $\dot{\delta}_{\epsilon}$ and $\delta_{\eta}^{\prime}$, respectively). Mapping (4.1) leads to the infinitesimal changes of the fields $X^{\mu}(\tau, \sigma)$ and $\Pi_{\mu}(\tau, \sigma)=\partial L / \partial \dot{X}^{\mu}=$ $\mu\left(\frac{1}{A} g_{\mu \nu} \dot{X}^{\nu}-\frac{A^{1}}{A} g_{\mu \nu} \dot{X}^{\nu}\right)$ as follows

$$
\delta X^{\mu}=\dot{\delta}_{\epsilon} X^{\mu}+\delta_{\eta}^{\prime} X^{\mu}=\epsilon \dot{X}^{\mu}+\eta \dot{X}^{\mu}, \quad \delta \Pi_{\mu}=\epsilon \dot{\Pi}_{\mu}+\dot{\epsilon}\left(A^{1} \Pi_{\mu}+\mu A g_{\mu \nu} \dot{X}^{\nu}\right)+\left(\eta \Pi_{\mu}\right)^{\prime}
$$


The transformations (4.2) are defined along curves in the phase space with coordinates $\left(X^{\mu}, \Pi_{\mu}\right)$ and are expected to be generated by the first-class constraints $\check{C}$ and $\check{C}_{1}$ according to the theory of gauge systems $[10,11]$. One verifies that

$$
\begin{aligned}
\left\{X^{\mu}, \check{C}(\varphi)\right\} & =\frac{\varphi}{\mu} \Pi_{\mu}, \quad\left\{\Pi_{\mu}, \check{C}(\varphi)\right\}=-\frac{\varphi}{2 \mu}\left(\Pi_{\alpha} \Pi_{\beta} g_{, X^{\mu}}^{\alpha \beta}+\dot{X}^{\alpha} \dot{X}^{\beta} g_{\alpha \beta, X^{\mu}}\right)+\mu\left(\varphi g_{\mu \nu} \dot{X}^{\nu}\right)^{\prime}, \\
\left\{X^{\mu}, \check{C}_{1}(\phi)\right\} & =\phi \dot{X}^{\mu}, \quad\left\{\Pi_{\mu}, \check{C}_{1}(\phi)\right\}=\left(\phi \Pi_{\mu}\right)^{\prime},
\end{aligned}
$$

where $\phi(\sigma, \tau)$ and $\varphi(\sigma, \tau)$ are smearing functions depending on two variables, and the integration defining the smearing of the constraints $C$ and $C_{1}$ does not include the integration with respect to $\tau$ variable (see (3.3)).

The comparison of (4.2) with (4.3)-(4.4) gives specific relations between these two transformations. For the action of the constraints along curves in the phase space, which are solutions to the equations of motion, we get

$$
\begin{aligned}
\left\{X^{\mu}, \check{C}(\varphi)\right\}=\dot{\delta}_{\frac{\varphi}{A}} X^{\mu}-\delta_{\frac{A^{1} \varphi}{A}}^{\prime} X^{\mu}, & \left\{\Pi_{\mu}, \check{C}(\varphi)\right\} & =\dot{\delta}_{\frac{\varphi}{A}} \Pi_{\mu}-\delta_{\frac{A^{1} \varphi}{A}}^{\prime} \Pi_{\mu}, \\
\left\{X^{\mu}, \check{C}_{1}(\phi)\right\}=\delta_{\phi}^{\prime} X^{\mu}, & \left\{\Pi_{\mu}, \check{C}_{1}(\phi)\right\} & =\delta_{\phi}^{\prime} \Pi_{\mu} .
\end{aligned}
$$

We see that the relation between gauge transformations in the string coordinate space and its phase space may be established only for curves (coordinate transformations are not time-invariant), which are solutions to the Hamilton equations. This is why the above relation depends on the specific choice of $A$ and $A^{1}$.

\section{Representations}

This section is devoted to the Dirac quantization of the system. It consists of two essential steps: (i) definition of a self-adjoint representation of the algebra of constraints on a kinematical Hilbert space, and (ii) solution to the constraints, i.e. finding the common domain on which all the constraint operators vanish, which is used to construct a physical Hilbert space.

It is clear that (3.10)-(3.12) consists of two independent subalgebras. To be specific, we first quantize the subalgebra satisfied by

$$
L_{n}:=\check{C}_{+}(\exp i n \sigma), \quad n \in \mathbb{Z} .
$$

The imposition of the constraint (5.1) with $n \in \mathbb{Z}$ (but not with $n \in \mathbb{N}$, that is specific to the conformal field theory quantization) is consistent since for the real fields $X^{\mu}$ and $\Pi_{\mu}$ holds $\overline{L_{n}}=L_{-n}$ and the constraints $L_{n}$ and $L_{-n}$ are equivalent. Moreover, including the complex conjugation for the classical constraints allows for introducing adjoint constraint operators at quantum level as we shall see later.

One may easily verify that

$$
\left\{L_{n}, L_{m}\right\}=i(m-n) L_{m+n} .
$$

Quantization of (3.11) can be done by analogy. Merger of both quantum subalgebras will complete the problem of finding the representation of the full algebra (3.10)-(3.12). To 
construct the representation of the algebra (3.10)-(3.12), which consists of two commuting subalgebras, one may use standard techniques [6,14]. For instance, the representation space of the algebra may be defined to be either a tensor product or direct sum of the representations of both subalgebras.

\subsection{Representation based on a single field}

\subsubsection{Hilbert space}

The pre-Hilbert space, $\tilde{\mathcal{H}}$, induced by the space of fields, $\mathbb{S} \ni \sigma \rightarrow X(\sigma)$, is defined to be

$$
\begin{aligned}
\tilde{\mathcal{H}} \ni \Psi[X] & :=\int \psi(X, \dot{X}, \sigma) d \sigma, \\
\langle\Psi \mid \Phi\rangle & :=\int \bar{\Psi}[X] \Phi[X][d X],
\end{aligned}
$$

where $\psi(X, \dot{X}, \sigma)$ is such that $\langle\Psi \mid \Psi\rangle\langle\infty$. The measure $[d X]$ is assumed to be invariant with respect to $\sigma$ reparametrization. Completion of $\tilde{\mathcal{H}}$ in the norm induced by (5.4) defines the Hilbert space $\mathcal{H}$.

\subsubsection{Representation of generator}

In what follows we find a representation of (5.2). Let us consider a diffeomorphism on $\mathbb{S}^{1}$ of the form $X(\sigma) \mapsto X(\sigma+\epsilon v(\sigma))$. For a small $\epsilon$ we have

$$
\begin{aligned}
& X(\sigma+\epsilon v(\sigma)) \approx X(\sigma)+\epsilon v(\sigma) \dot{X}(\sigma)=: X(\sigma)+\epsilon L_{v} X(\sigma), \\
& \dot{X}(\sigma+\epsilon v(\sigma)) \approx \dot{X}(\sigma)+\epsilon \frac{d}{d \sigma}[v(\sigma) \dot{X}(\sigma)]=\dot{X}(\sigma)+\epsilon \frac{d}{d \sigma}\left[L_{v} X(\sigma)\right] .
\end{aligned}
$$

Now, we define an operator $\hat{L}_{v}$ corresponding to $L_{v}$ defined by (5.5). Since we have

$$
\Psi[X(\sigma+\epsilon v(\sigma))] \approx \Psi[X(\sigma)]+\epsilon \int\left(\frac{\partial \psi}{\partial X} L_{v} X+\frac{\partial \psi}{\partial \dot{X}} \frac{d}{d \sigma}\left[L_{v} X\right]\right) d \sigma
$$

we set

$$
\hat{L}_{v} \Psi[X]:=\int\left(\frac{\partial \psi}{\partial X} L_{v} X+\frac{\partial \psi}{\partial \dot{X}} \frac{d}{d \sigma}\left[L_{v} X\right]\right) d \sigma=\int\left(\dot{v} \frac{\partial \psi}{\partial \dot{X}} \dot{X}-\dot{v} \psi-v \frac{\partial \psi}{\partial \sigma}\right) d \sigma \in \mathcal{H} .
$$

One may verify that $\left\{L_{v}, L_{w}\right\}=L_{(v \dot{w}-\hat{v} w)}$ and check that

$$
\left[\hat{L}_{v}, \hat{L}_{w}\right]=\hat{L}_{(v \dot{w}-\dot{v} w)} .
$$

Next, let us consider the following

$$
\begin{aligned}
\int \bar{\Psi}[X(\sigma+\epsilon v(\sigma))] \Phi[X(\sigma)][d X(\sigma)] & =\int \bar{\Psi}[X(\sigma)] \Phi[X(\sigma-\epsilon v(\sigma))][d X(\sigma-\epsilon v(\sigma))] \\
& =\int \bar{\Psi}[X(\sigma)] \Phi[X(\sigma-\epsilon v(\sigma))][d X(\sigma)]
\end{aligned}
$$

where we assume that $v(\sigma)$ is a real function and $\sigma \mapsto \sigma+\epsilon v(\sigma)$ is a diffeomorphism. Taking derivative with respect to $\epsilon$ of both sides of (5.10) and putting $\epsilon=0$ leads to

$$
\left\langle\hat{L}_{v} \Psi \mid \Phi\right\rangle=-\left\langle\Psi \mid \hat{L}_{v} \Phi\right\rangle
$$


Therefore, the real and imaginary parts of the operator $\hat{L}_{n}$ defined by the mapping

$$
L_{n} \mapsto \hat{L}_{n}:=i \hbar \hat{L}_{\exp (i n \sigma)}
$$

are symmetric on $\mathcal{H}$ and lead to a symmetric representation of the algebra (5.2), which now reads

$$
\frac{1}{i \hbar}\left[\hat{L}_{n}, \hat{L}_{m}\right]=i(m-n) \hat{L}_{m+n}
$$

The equation (5.13) is a self-adjoint representation of (5.2) if $\hat{L}_{n}$ are bounded operators [9].

\subsubsection{Solving the constraint}

Since we look for diffeomorphism invariant states, it is sufficient to assume that $\psi=$ $\psi(X, \dot{X})$. Let us solve the equation

$$
\hat{L}_{n} \Psi=0,
$$

which after making use of (5.8) and integrating by parts reads

$$
\int\left(e^{i n \sigma}\right)\left[-\psi+\frac{\partial \psi}{\partial \dot{X}} \dot{X}\right] d \sigma=0 .
$$

General solution to (5.15) has the form

$$
-\psi+\frac{\partial \psi}{\partial \dot{X}} \dot{X}=\sum_{k \neq-n} a_{k} e^{\imath k \sigma} \quad \text { for } \quad n \neq 0
$$

where $a_{k}$ are arbitrary constants, and there is no condition for $n=0$. Our goal is an imposition of all the constraint, i.e. we look for $\Psi: \forall n \hat{L}_{n} \Psi=0$. We find that the intersection of all the kernels defined by (5.16) is given by the equation

$$
-\psi+\frac{\partial \psi}{\partial \dot{X}} \dot{X}=c
$$

where $c$ is an arbitrary constant. It is enough to solve (5.17) for $c=0$ and then simply add to the solution any constant. Since the above equation results from (5.15), it is expected to hold in a more general sense, i.e. in a distributional sense. It is clear that the space of solutions to (5.17) is defined by

$$
\psi=\alpha(X)|\dot{X}|+\beta(X) \dot{X}-c
$$

where $\alpha$ and $\beta$ are any functions. The first term is a distribution, the second one can be checked to be trivial, since

$$
\int_{\mathbb{S}^{1}} \beta(X) \dot{X} d \sigma=\int_{\mathbb{S}^{1}} \beta(X) d X=0
$$

for a periodic field $X$, and third one is a functional that gives the same value $2 \pi c$ for every field.

Notice that taking $n>0$ (as in the conformal field theory) would reduce (5.16) to the form

$$
-\psi+\frac{\partial \psi}{\partial \dot{X}} \dot{X}=\sum_{k \neq-n} a_{k} e^{\imath k \sigma} \quad \text { for } \quad n>0
$$


and subsequently would lead to the solution of (5.20) in the form

$$
\psi=\alpha(X)|\dot{X}|+\beta(X) \dot{X}-\sum_{k>-1} a_{k} e^{\imath k \sigma},
$$

instead of (5.17), which is not diffeomorphism invariant due to the last term. The diffeomorphism is the basic symmetry underlying our paper.

\subsubsection{Interpretation of solutions}

Let us identify special features of the fields $X$ evaluated through the first term in (5.21)

$$
\begin{aligned}
\Psi[X] & =\int \alpha(X)|\dot{X}| d \sigma=\int \frac{d}{d \sigma}[\gamma(X)](\tilde{H}(\dot{X})-\tilde{H}(-\dot{X})) d \sigma \\
& =-\int \gamma(X) 2 \delta(\dot{X}) d \dot{X}=-\sum_{\operatorname{extr} X} 2 \gamma(X)=\sum_{\min X} 2 \gamma(X)-\sum_{\max X} 2 \gamma(X)
\end{aligned}
$$

where $d \gamma / d X=\alpha$ and $\tilde{H}$ is the Heaviside function. Thus, $\Psi$ depends on the values of $\gamma$ at extrema points of $X$. We have diffeomorphism invariance due to the implication $\left(\frac{d X}{d \sigma}=0\right) \Rightarrow\left(\frac{d X}{d \tilde{\sigma}}=\frac{d \sigma}{d \widetilde{\sigma}} \frac{d X}{d \sigma}=0\right)$.

\subsection{Representation based on phase space functions}

\subsubsection{Hilbert space}

Using the ideas with the single field case (presented in the previous subsection), we try construct now the representation of the algebra (3.10)-(3.12) by making use of the phase space functions with coordinates $\left(X^{\mu}, \Pi_{\mu}\right)$, where $\mu=0,1, \ldots, d-1$.

As before, we propose to include fields $\mathbb{S} \ni \sigma \rightarrow Y^{\mu}(\sigma)$ as well as their first derivatives $\bar{Y}^{\mu}(\sigma)$ in the definition of a state

$$
\begin{aligned}
\mathcal{H} \ni \Psi \vec{Y}] & :=\int \psi(\vec{Y}, \dot{\vec{Y}}, \sigma) d \sigma, \\
\langle\Psi \mid \Phi\rangle & :=\int \bar{\Psi}[\vec{Y}] \Phi[\vec{Y}][d \vec{Y}],
\end{aligned}
$$

where $\vec{Y} \equiv\left(Y^{\mu}\right)$, and where $\psi(\vec{Y}, \dot{\vec{Y}}, \sigma)$ is any well-behaved function such that $\langle\Psi \mid \Psi\rangle<\infty$.

Inspired by [18], we try to find two sets of phase space functions $Y_{ \pm}$by the requirement that they Poisson commute with the constraints:

$$
\begin{aligned}
0=\left\{C_{ \pm}(u), Y_{ \pm}(v)\right\}= & \int-\left[\frac{d}{d \sigma}\left(\frac{u}{2} \kappa T \dot{X}_{\nu}\right) \pm \frac{d}{d \sigma}\left(\frac{u}{2} \Pi_{\nu}\right)\right] \frac{\delta Y_{ \pm}(v)}{\delta \Pi_{\nu}} \\
& +\left[-\frac{u}{4} \frac{\Pi^{2}}{\kappa T^{2}}+\frac{u}{4} \kappa \dot{X}^{2}\right] \frac{\delta Y_{ \pm}(v)}{\delta \Pi_{0}}-\left[\frac{u}{2} \frac{\Pi^{\nu}}{\kappa T} \pm \frac{u}{2} \dot{X}^{\nu}\right] \frac{\delta Y_{ \pm}(v)}{\delta X^{\nu}} d \sigma .
\end{aligned}
$$

The indices are lowered or raised by $\eta_{\mu \nu}$ or $\eta^{\mu \nu}$, respectively, and

$$
\frac{\delta Y_{ \pm}(v)}{\delta Z}:=\Sigma_{n=0}^{\infty}(-1)^{n}\left(\frac{\partial Y_{ \pm}(v)}{\partial Z^{(n)}} v\right)^{(n)}
$$

where $(\ldots)^{(n)}$ denotes the n-th derivative with respect to $\sigma$. 
Finding solution to eq. (5.25) determines two domains of definition of quantum states. Imposition of a constraint on each of them defines two physical phase spaces. Hilbert space of the whole system may be defined, for instance, as a direct sum or a tensor product of these two Hilbert spaces.

Let us introduce new coefficients

$$
D_{ \pm}^{\nu}:=\frac{1}{2} \Pi^{\nu} \pm \frac{1}{2} \kappa T \dot{X}^{\nu}
$$

so that (5.25), after taking into account (5.26), reads

$$
\begin{aligned}
\left\{C_{ \pm}(u), Y_{ \pm}(v)\right\}= & \int v \Sigma_{n=0}^{\infty}\left[\mp\left(u D_{ \pm}^{\nu}\right)^{(n+1)} \frac{\partial Y_{ \pm}}{\partial\left(\Pi^{\nu}\right)^{(n)}}+\left(u \frac{D_{+}^{\mu} D_{-\mu}}{\kappa T^{2}}\right)^{(n)} \frac{\partial Y_{ \pm}}{\partial\left(\Pi^{0}\right)^{(n)}}\right. \\
& \left.-\left(u \frac{D_{ \pm}^{\nu}}{\kappa T}\right)^{(n)} \frac{\partial Y_{ \pm}}{\partial\left(X^{\nu}\right)^{(n)}}\right] d \sigma .
\end{aligned}
$$

The condition $\left\{C_{ \pm}(u), Y_{ \pm}(v)\right\}=0$ must hold for any $v$, hence

$$
\Sigma_{n=0}^{\infty}\left[\mp\left(u D_{ \pm}^{\nu}\right)^{(n+1)} \frac{\partial Y_{ \pm}}{\partial\left(\Pi^{\nu}\right)^{(n)}}+\left(u \frac{D_{+}^{\mu} D_{-\mu}}{\kappa T^{2}}\right)^{(n)} \frac{\partial Y_{ \pm}}{\partial\left(\Pi^{0}\right)^{(n)}}-\left(u \frac{D_{ \pm}^{\nu}}{\kappa T}\right)^{(n)} \frac{\partial Y_{ \pm}}{\partial\left(X^{\nu}\right)^{(n)}}\right]=0 .
$$

As $u$ is arbitrary too, we obtain the following infinite set of equations:

$$
\begin{array}{r}
\sum_{n=m}^{\infty} \frac{n !}{(n-m) !}\left[\mp\left(D_{ \pm}^{\nu}\right)^{(n-m)} \frac{\partial Y_{ \pm}}{\partial\left(\Pi^{\nu}\right)^{(n-1)}}+\left(\frac{D_{+}^{\mu} D_{-\mu}}{\kappa T^{2}}\right)^{(n-m)} \frac{\partial Y_{ \pm}}{\partial\left(\Pi^{0}\right)^{(n)}}\right. \\
\left.-\left(\frac{D_{ \pm}^{\nu}}{\kappa T}\right)^{(n-m)} \frac{\partial Y_{ \pm}}{\partial\left(X^{\nu}\right)^{(n)}}\right]=0
\end{array}
$$

where $m=0,1 \ldots$

It is very difficult to solve the system (5.30) in its full generality. Let us assume for simplicity that $Y_{ \pm}=\tilde{Y}_{ \pm}:=Y_{ \pm}(X, \dot{X}, \Pi)$, i.e. we ignore possible dependance on higher derivatives of $X$ and $\Pi$ with respect to $\sigma$. In such a case an infinite system of equations (5.30) simplifies to the following system of only two equations:

$$
\begin{array}{r}
D_{ \pm}^{\nu} \frac{\partial \tilde{Y}}{\partial D_{ \pm}^{\nu}}=0, \\
\mp 2 D_{ \pm}^{\nu} \frac{\partial \tilde{Y}}{\partial D_{ \pm}^{\nu}}+\frac{1}{\kappa T^{2}} D_{-\nu} D_{+}^{\nu}\left(\frac{\partial \tilde{Y}}{\partial D_{+}^{0}}+\frac{\partial \tilde{Y}}{\partial D_{-}^{0}}\right)-2 D_{ \pm}^{\nu} \frac{1}{\kappa T} \frac{\partial \tilde{Y}}{\partial X^{\nu}} \\
-D_{ \pm}^{0} \frac{1}{\kappa T^{2}}\left(D_{+}^{\nu}-D_{-}^{\nu}\right)\left(\frac{\partial \tilde{Y}}{\partial D_{+}^{\nu}}-\frac{\partial \tilde{Y}}{\partial D_{-}^{\nu}}\right) \mp \frac{1}{\kappa T^{2}}\left(D_{+}^{0}-D_{-}^{0}\right) D_{ \pm}^{\nu} \frac{\partial \tilde{Y}}{\partial D_{\mp}^{\nu}}=0 .
\end{array}
$$

Due to the assumption that $\tilde{Y}_{ \pm}$does not depend neither on $\dot{D}_{+}^{\nu}$ nor on $\dot{D}_{-}^{\nu}$, we obtain that the solution to (5.31) reads $\tilde{Y}_{ \pm}=\tilde{Y}_{ \pm}\left(X^{\mu}, D_{\mp}^{\mu}\right)$. Thus, eq. (5.32) turns into

$$
\begin{gathered}
\frac{1}{\kappa T^{2}} D_{-\nu} D_{+}^{\nu} \frac{\partial \tilde{Y}_{ \pm}}{\partial D_{\mp}^{0}}-2 D_{ \pm}^{\nu} \frac{1}{\kappa T} \frac{\partial \tilde{Y}_{ \pm}}{\partial X^{\nu}} \\
\pm D_{ \pm}^{0} \frac{1}{\kappa T^{2}}\left(D_{+}^{\nu}-D_{-}^{\nu}\right) \frac{\partial \tilde{Y}_{ \pm}}{\partial D_{\mp}^{\nu}} \mp \frac{1}{\kappa T^{2}}\left(D_{+}^{0}-D_{-}^{0}\right) D_{ \pm}^{\nu} \frac{\partial \tilde{Y}_{ \pm}}{\partial D_{\mp}^{\nu}}=0 .
\end{gathered}
$$


Since $\tilde{Y}_{ \pm}$does not depend on $D_{\mp}^{\nu}$, we conclude that eq. (5.33) splits into the following system of equations:

$$
\begin{aligned}
D_{\mp}^{i} \frac{\partial \tilde{Y}_{ \pm}}{\partial D_{\mp}^{0}}- & 2 T \frac{\partial \tilde{Y}_{ \pm}}{\partial X^{i}}+D_{\mp}^{0} \frac{\partial \tilde{Y}_{ \pm}}{\partial D_{\mp}^{i}}=0, \quad \text { for } \quad i=1, \ldots, d \\
- & 2 T \frac{\partial \tilde{Y}_{ \pm}}{\partial T}-D_{\mp}^{\nu} \frac{\partial \tilde{Y}_{ \pm}}{\partial D_{\mp}^{\nu}}=0 .
\end{aligned}
$$

Equations (5.34) and (5.35) have only two independent solutions:

$$
\tilde{Y}_{ \pm}=\frac{D_{\mp}^{\mu} D_{\mp \mu}}{\kappa T}=C_{\mp} .
$$

Such solutions have been expected due to $\left\{C_{+}(u), C_{-}(v)\right\}=0$, for any $u$ and $v$. We have shown that these are the only solutions to (5.25) under the assumption that $Y_{ \pm}=$ $Y_{ \pm}(X, X, \Pi)$. More general solutions may be found by admitting that $Y_{ \pm}$depend on higher derivatives of $X$ and $\Pi$. Considering of such generalizations is, however, beyond the scope of the present paper.

\subsubsection{Solving the constraint}

Now, let us make use the solution to eq. (5.25) for each region separately. We assume that $\psi=\psi(\vec{Y}, \dot{\vec{Y}})$. Let us solve the equation

$$
\hat{L}_{n} \Psi[\vec{Y}]=0,
$$

which in the case of many fields is a simple extension of (5.15), and reads

$$
\int\left(e^{i n} \sigma\right)\left[-\psi+\frac{\partial \psi}{\partial \dot{Y}^{\mu}} \dot{Y}^{\mu}\right] d \sigma=0
$$

By analogy to the single field case we infer that

$$
-\psi+\frac{\partial \psi}{\partial \dot{Y}^{\mu}} \dot{Y}^{\mu}=\sum_{k \neq-n} a_{k} e^{\imath k \sigma} \quad \text { for } n \neq 0
$$

and again with no condition for $n=0$. Imposing all the constraints leads to

$$
-\psi+\frac{\partial \psi}{\partial \dot{Y}^{\mu}} \dot{Y}^{\mu}=c .
$$

One can check that the solutions form a linear space and are of the form

$$
\psi=\left(\sum_{i} \alpha_{i}(\vec{Y}) \prod_{\mu}\left|\dot{Y}^{\mu}\right|^{\rho_{i}^{\mu}}\right)^{\frac{1}{\rho}}-c
$$

where $\sum_{\mu} \rho_{i}^{\mu}=\rho$. This is an expected result since the measure $\sqrt[f]{\prod_{\mu}\left|Y^{\mu}\right|^{\rho^{\mu}}} d \sigma$ is invariant with respect to $\sigma$-diffeomorphisms. 


\subsubsection{Interpretation of solutions}

Suppose we have a space $V \ni \vec{Y}$ in which a closed curve, $\sigma \mapsto Y^{\mu}(\sigma)$, is embedded. Due to (5.41) we have a kind of measure in $V$ given by

$$
\sqrt[\rho]{\alpha(\vec{Y}) \prod_{\mu}\left|d Y^{\mu}\right| \rho^{\mu}}
$$

One may say, it is a generalization of the Riemannian type metric, since for $\rho_{i}^{\mu}=1$ and $\rho=2$ we have

$$
\sqrt{g_{\mu \nu} d Y^{\mu} d Y^{\nu}}
$$

where $g_{\mu \nu}=g_{\mu \nu}(\vec{Y})$. In the case, e.g., $Y^{0}$ is not a constant field, (5.42) becomes

$$
\sqrt{\alpha(\vec{Y}) \prod_{\mu}\left|d Y^{\mu}\right| \rho^{\mu}}=\sqrt[\rho]{\alpha(\vec{Y}) \prod_{\mu \neq 0}\left|\frac{d Y^{\mu}}{d Y^{0}}\right|^{\rho^{\mu}}}\left|d Y^{0}\right|=: \widetilde{\alpha}\left(Y^{0}\right)\left|d Y^{0}\right| .
$$

Thus, it is an extension of the single field metric defined by (5.22), which may be rewritten as $\alpha(Y)|d Y|$. In this case however integration (5.44) is performed in the multidimensional space so $\widetilde{\alpha}\left(Y^{0}\right)$ depends on a particular curve (not just its end points). In fact, it is a measure of relative variation of fields, i.e. quantity that is both gauge-invariant and determines curve uniquely. Two simple examples of wavefunction for two fields $Y_{1}$ and $Y_{2}$ are given by

$$
\begin{aligned}
& \psi=\alpha\left(Y_{1} \pm Y_{2}\right)\left|\dot{Y}_{1} \pm \dot{Y}_{2}\right|, \\
& \psi=\alpha\left(Y_{1} Y_{2}\right)\left|\dot{Y}_{1} Y_{2}+Y_{1} \dot{Y}_{2}\right|,
\end{aligned}
$$

where in analogy to the single field case, (5.45) and (5.46) 'measure extrema points' for fields $Y_{1} \pm Y_{2}$ and $Y_{1} Y_{2}$, respectively.

It is clear that finding the representation of the complete algebra (3.10)-(3.12), may be carried out by analogy to the single field case by using standard techniques $[6,14]$. For instance, we may define $\Psi\left[Y_{+}^{\mu}, Y_{-}^{\mu}\right]:=\Psi\left[Y_{+}^{\mu}\right] \otimes \Psi\left[Y_{-}^{\mu}\right]$.

\subsection{Remarks on representations of observables}

In the space of solutions to the constraints there are many types of measures in the form (5.42) which may be used to define a variety of physical Hilbert spaces and representations. One may associate operators, in physical Hilbert space, with homeomorphisms $V \mapsto V$. The operators split the Hilbert space into a set of invariant subspaces, each of which defines a specific representation. Each subspace is connected with specific measure and all other measures that are produced by homeomorphisms. For example, the products of the action of homeomorphism upon a metric (of Riemannian manifold) constitute the space of all the metrics that are equivalent modulo a change of coordinates and all other metrics that are reductions of the initial metric.

Now, let us consider an infinitesimal homeomorphism, $\widehat{O}_{u}: V \rightarrow V$, of the space $V$ along the vector field $u=u^{\lambda}(\vec{Y}) \partial / \partial Y^{\lambda}$. In what follows we consider an example of representation: 
For the special form of (5.41) defined by

$$
\psi:=\alpha_{\mu}(\vec{Y}) \dot{Y}^{\mu}, \quad \text { or } \quad \Psi[Y]=\int \alpha_{\mu}(\vec{Y}) d Y^{\mu}
$$

we find that [23]

$$
\frac{1}{i \hbar} \widehat{O}_{u}\left(\int \alpha_{\mu} d Y^{\mu}\right)=\int\left(u^{\lambda} \alpha_{\mu, \lambda}+u_{, \mu}^{\lambda} \alpha_{\lambda}\right) d Y^{\mu} .
$$

One may verify that the operators $\widehat{O}_{u}$ and $\widehat{O}_{v}$ associated with vector fields $u$ and $v$ satisfy the algebra

$$
\frac{1}{i \hbar}\left[\widehat{O}_{u}, \widehat{O}_{v}\right]=\widehat{O}_{[u, v]}
$$

The representation defined by (5.48) and (5.49) is self-adjoint if the operators are bounded.

\section{Conclusions}

The quantization problem of a membrane embedded in a time dependent orbifold is difficult. It has not been solved satisfactory so far even for the case of the Minkowski target space (see, eg. [15]). Most proposals for quantum theory of membranes are based on finding relationships between very special membrane states and string states (see, e.g. $[16,17]$ ). In this paper we have considered states of membrane winding uniformly around compact dimension of the background space.

An action integral of a membrane winding uniformly around compact dimension of $\mathrm{CM}$ space depends on functions of two variables and there are only two constraints. The dynamics of a membrane, with the parameters space topology $\mathbb{S}^{1} \times \mathbb{S}^{1}$, winding uniformly around compact dimension of embedding spacetime is mathematically equivalent to the dynamics of a closed string in a flat FRW spacetime.

General quantum theory of a string in a curved spacetime has not been constructed yet. We have proposed a framework which includes curved background and is based on Dirac's quantization of the diffeomorphism symmetry. However, it is different from the quantization program initiated in [18].

The first-class constraints specifying the dynamics of a membrane propagating in the compactified Milne space satisfy the algebra which is a Poisson algebra. Methods for finding a self-adjoint representation of such type of an algebra are complicated [13]. We overcome this difficulty by the reduction and redefinition of the constraints algebra. Resulting algebra is a Lie algebra which simplifies the problem of quantization of the membrane dynamics.

We found an example of solution to eq. (5.25) which enables defining the Hilbert space of the system. The imposition of the constraints led to the physical states. It means that the singularity of the CM space is not an insurmountable obstacle for the construction of a quantum model of a system describing propagation of a membrane. There exist non-trivial states of a membrane evolving across the singularity. Quantum states of a membrane winding uniformly around compact dimension of the CM space are examples of such nontrivial states. There may exist the membrane states which cannot be quantized by our method. We postpone an examination of this issue to our next papers. 
The quantum membrane completes our preliminary tests of the 'transparency' of the cosmological singularity of the cyclic model of the universe: there exist non-trivial quantum states of $p$-branes (particle, string, membrane) that can propagate from the pre-singularity to the post-singularity epoch. Extended objects may 'cure' the disappearance of the compact dimension at the singularity, which is specific to the time dependent orbifolds. The propagation of a particle across the singularity may be indeterministic, whereas higher dimensional objects seem to propagate uniquely. The big-crunch/big-bang model of the universe with quantum objects propagating in classical spacetime may make sense. However, this is not the end of the story. We have not examined all possible states. There may exist quantum $p$-brane states which may lead to problems at the singularity and should be analyzed.

There is still another problem to be examined in the context of the cyclic model: We have considered so far the propagation of test $p$-branes (i.e. objects which do not modify the background space). The $p$-branes which are physical may lead to the gravitational instability. It has been argued [19] that due to this problem the big-crunch of the cyclic model may collapse into a black hole which would end the evolution of the universe. In such a case, the cyclic model scenario would need to be modified to make sense. One may study this problem by quantization of the entire system, i.e. $p$-brane and the embedding spacetime, by making use of the loop quantum cosmology. We have already made some preliminary steps to examine this problem $[21,22]$.

\section{Acknowledgments}

This work has been supported by the Polish Ministry of Science and Higher Education Grant NN 202054233.

\section{A Local form of the constraints algebra}

It has been found (see appendix 2 of [16]) that the constraints (3.1) and (3.2) satisfy the algebra

$$
\begin{aligned}
\left\{C(\sigma), C\left(\sigma^{\prime}\right)\right\} & =8 \kappa^{2} T^{2}(\sigma) C_{1}(\sigma) \frac{\partial}{\partial \sigma} \delta\left(\sigma^{\prime}-\sigma\right)+4 \kappa^{2} \delta\left(\sigma^{\prime}-\sigma\right) \frac{\partial}{\partial \sigma}\left(T^{2}(\sigma) C_{1}(\sigma)\right), \\
\left\{C(\sigma), C_{1}\left(\sigma^{\prime}\right)\right\} & =2 C(\sigma) \frac{\partial}{\partial \sigma} \delta\left(\sigma^{\prime}-\sigma\right)+\delta\left(\sigma^{\prime}-\sigma\right) \frac{\partial}{\partial \sigma} C(\sigma), \\
\left\{C_{1}(\sigma), C_{1}\left(\sigma^{\prime}\right)\right\} & =2 C_{1}(\sigma) \frac{\partial}{\partial \sigma} \delta\left(\sigma^{\prime}-\sigma\right)+\delta\left(\sigma^{\prime}-\sigma\right) \frac{\partial}{\partial \sigma} C_{1}(\sigma),
\end{aligned}
$$

where $\partial X^{\mu}\left(\sigma^{\prime}\right) / \partial X^{\nu}(\sigma)=\delta_{\nu}^{\mu} \delta\left(\sigma^{\prime}-\sigma\right)=\partial \Pi_{\nu}\left(\sigma^{\prime}\right) / \partial \Pi_{\mu}(\sigma)$ (with other partial derivatives being zero), and where the Poisson bracket is defined to be

$$
\{\cdot, \cdot\}:=\int_{-\pi}^{\pi} d \sigma\left(\frac{\partial \cdot}{\partial X^{\mu}} \frac{\partial \cdot}{\partial \Pi_{\mu}}-\frac{\partial \cdot}{\partial \Pi_{\mu}} \frac{\partial \cdot}{\partial X^{\mu}}\right) .
$$

Open Access. This article is distributed under the terms of the Creative Commons Attribution Noncommercial License which permits any noncommercial use, distribution, and reproduction in any medium, provided the original author(s) and source are credited. 


\section{References}

[1] P.J. Steinhardt and N. Turok, A cyclic model of the universe, hep-th/0111030 [SPIRES].

[2] P.J. Steinhardt and N. Turok, Cosmic evolution in a cyclic universe, Phys. Rev. D 65 (2002) 126003 [hep-th/0111098] [SPIRES].

[3] J. Khoury, B.A. Ovrut, N. Seiberg, P.J. Steinhardt and N. Turok, From big crunch to big bang, Phys. Rev. D 65 (2002) 086007 [hep-th/0108187] [SPIRES].

[4] P. Malkiewicz and W. Piechocki, Toy model of big-crunch/big-bang transition, Class. Quant. Grav. 23 (2006) 2963 [gr-qc/0507077] [SPIRES].

[5] P. Malkiewicz and W. Piechocki, Probing the cosmic singularity with a particle, Class. Quant. Grav. 23 (2006) 7045 [gr-qc/0606091] [SPIRES].

[6] P. Malkiewicz and W. Piechocki, Propagation of a string across the cosmic singularity, Class. Quant. Grav. 24 (2007) 915 [gr-qc/0608059] [SPIRES].

[7] P. Malkiewicz and W. Piechocki, Excited states of a string in time dependent orbifold, Class. Quant. Grav. 26 (2009) 015008 [arXiv:0807.2990] [SPIRES].

[8] O. Milbredt, The Cauchy Problem for Membranes, arXiv:0807.3465 [SPIRES].

[9] M. Reed and B. Simon, Methods of modern mathematical physics, Academic Press, New York U.S.A. (1975).

[10] P.A.M. Dirac, Lectures on quantum mechanics, Belfer Graduate School of Science Monographs Series, New York U.S.A. (1964).

[11] M. Henneaux and C. Teitelboim, Quantization of gauge systems Princeton University Press, Princeton U.S.A. (1992).

[12] J. Polchinski, String theory, Cambridge University Press, Cambridge U.K. (1998).

[13] T. Thiemann, Modern canonical quantum general relativity, Cambridge University Press, Cambridge U.K. (2007).

[14] E. Prugovečki Quantum mechanics in Hilbert space, Academic Press, New York U.S.A. (1981).

[15] L. Smolin, Covariant quantization of membrane dynamics, Phys. Rev. D 57 (1998) 6216 [hep-th/9710191] [SPIRES].

[16] N. Turok, M. Perry and P.J. Steinhardt, M theory model of a big crunch /big bang transition, Phys. Rev. D 70 (2004) 106004 [hep-th/0408083] [SPIRES].

[17] P. Hořava, Membranes at quantum criticality, JHEP 03 (2009) 020 [arXiv:0812.4287] [SPIRES].

[18] T. Thiemann, The LQG string: Loop quantum gravity quantization of string theory. I: Flat target space, Class. Quant. Grav. 23 (2006) 1923 [hep-th/0401172] [SPIRES].

[19] G.T. Horowitz and J. Polchinski, Instability of spacelike and null orbifold singularities, Phys. Rev. D 66 (2002) 103512 [hep-th/0206228] [SPIRES].

[20] P. Malkiewicz and W. Piechocki, Energy scale of the big bounce, Phys. Rev. D 80 (2009) 063506 [arXiv:0903.4352] [SPIRES].

[21] P. Dzierzak, P. Malkiewicz and W. Piechocki, Turning big bang into big bounce. 1. Classical dynamics, Phys. Rev. D 80 (2009) 104001 [arXiv:0907.3436] [SPIRES]. 
[22] P. Malkiewicz and W. Piechocki, Turning big bang into big bounce: II. quantum dynamics, arXiv:0908. 4029 [SPIRES].

[23] A. Trautman Differential geometry for physicists, Stony Brook Lectures, (1984). 